Article

\title{
Ang mga Dialohikal na Hermenyutika nina Heidegger, Bultmann, at Gadamer Bilang Batayang Teoretikal sa Araling Filipino
}

\section{F. P. A. Demeterio}

\begin{abstract}
This paper is a study on the dialogical hermeneutics of Martin Heidegger, Rudolf Bultmann and Hans-Georg Gadamer, with the purview of extracting valuable lessons and uses, in terms of viable theories and frameworks, for the emerging discourses in Philippine Studies. It explores the hermeneutic theory of Heidegger by foregrounding his ontological theory of understanding. It unbundles the exegetical systems of Bultmann by tackling his form criticism, existential hermeneutics and demythologization theory. It discusses the speculations of Gadamer by starting with his understanding of prejudice, by moving on to his idea of the dialogical structure of understanding, and finally to the ironies behind his famous title "Truth and Method." In the end, this paper discusses in detail six lessons and uses from these hermeneutical theories for the benefit of Philippine Studies. Written in the Filipino language, this paper also desires to contribute to the Intellectualization of the Filipino language and the Filipinization of philosophical speculations in this country.
\end{abstract}

Keywords: Heidegger, Bultmann, Gadamer, dialogical hermeneutics

\section{Pundasyon ng Hermenyutika ayon kay Heidegger}

i Martin Heidegger (1889-1976), isang Alemang pilosopo at pilolohista, ang siyang nagpasimunong gawin ang hermenyutika bilang isang sentral na paksa sa pilosopiya. Para sa kanya, hindi lamang isang metodo ang hermenyutika, kung hindi ito mismo ang estraktura ng pag-uunawa ng tao. Sa puntong ito sisilipin muna natin ng ilang sandali ang aspekto ng hermenyutika bilang teorya para maintindihan natin ang pundasyon ng hermenyutika bilang metodo ni Heidegger. Metapisikal ang paksang pinagmumulan niya na nakatuon

(C) 2012 F.P.A. Demeterio

http://www.kritike.org/journal/issue 11/demeterio june2012.pdf

ISSN 1908-7330

\section{(cc) BY-NC}


sa masalimuot na usapin ng pagkakaiba ng Being (pag-iiral ng anumang bagay) at being (pagiging isang bagay).

Bukod sa mas pandiwa, o verb, ang Being habang mas bagay, o noun, ang being, naniniwala si Heidegger na mas mahalaga ang mas naunang tanong ng mga Presokratikong pantas kung ano nga ba kahulugan ng Being (pag-iiral ng anumang bagay) kumpara sa tanong kung ano ang depinisyon at mga katangian ng being (pagiging isang bagay). Ayon sa kanya naisasantabi ng kanluraing pilosopiya ang mas mahalagang tanong tungkol sa Being mula pa noong panahon nina Platon (428-347 BC) at Aristoteles (384-322 BC) dahil naging abala ang kanluraning pilosopiya sa tanong tungkol sa being. Sinabi ni Heidegger: "Ang tanong tungkol sa pag-iiral ng anumang bagay (Being) ay hindi makakarating sa kanyang wastong pagkakakongkreto hanggang hindi natin matatapos ang proseso ng pagbaklas ng ontolohikal na tradisyon (ang pagtatanong tungkol sa pagiging isang bagay, o being)."1

Para masusuri niya ang mahalagang tanong tungkol sa Being (pag-iiral ng anumang bagay) naghanap siya ng isang katangi-tanging being (bagay) para magsisilbing pintuan na maaaring pasukin ng kanyang proyekto; at ang napili niya ay walang iba kung hindi ang buhay ng tao, na tinatawag niyang Dasein. "Ang buhay ng tao (Dasein)," ipinaliwanag ni Heidegger, "ay hindi lamang isang bagay na katulad sa ibang mga bagay. Sa halip ito ay nabubukod-tangi bilang bagay dulot sa katotohanan na ang kanyang pag-iiral (Being) mismo ay kanyang itinatanong" (Heidegger, 32). Sa mas madaling salita, para masasagot niya ang tanong kung ano ang ibig sabihin ng pag-iiral ng anumang bagay (Being) dapat niya munang sagutin ang tanong kung ano ang ibig sabihin ng pag-iiral (Being) ng buhay ng tao (Dasien). Sa lahat ng mga bagay (being) tanging ang tao lamang ang mayroon, o maaaring magkakaroon, ng kamulatan tungkol sa kanyang sariling pag-iiral (Being) Para naman masasagot niya ang tanong kung ano ang ibig sabihin ng pag-iiral (Being) ng buhay ng tao (Dasein) humugot siya ng mga pilosopikal na kaisipan mula sa Griyegong si Platon at sa Danes na si Sóren Kierkegaard (1813-1855). Sa mistikal na antropolohiya ni Platon pinaniniwalaang nagmumula ang tao sa eternal at banal na prinsipyo. Ngunit sa kanyang pagsilang sa mundong ito nadungisan ang kanyang eternal at banal na kaluluwa at nagreresulta sa kanyang pagkalimut sa kanyang pinanggagalingang kaluwalhatian. Ang pagkakababad ng kaluluwa sa maruming materya ng mundo ay siyang nagbibigay ng lamat at kahinaan nito. Ito ang katumbas sa Kristiyanong konsepto ng nalaglag na kalikasan (fallen nature) ng tao. Para kay Platon, matatamo lamang ang kaligtasan ng tao sa pamamagitan ng kanyang pagmunimuni at pag-alaala sa kaluwalhatiang kanyang pinagmumulan (tingnan ang hugis 1). Mahalaga ang modelo ni Platon para kay Heidegger dahil nagpappakita ito kung papaano dapat iiral ang buhay ng tao sa loob ng panahon,

${ }^{1}$ Martin Heidegger, Being and Time (New York: Harper and Row, 1962), 49. 
mula sa kanyang pinanggalingan patungo sa kanyang papupuntahan. "Ang panahon," bigay diin ni Heidegger, "ay dapat maisama sa pagtatalakay-at mabalangkas ng husto-bilang konteksto sa lahat ng pag-iiral (Being) at ng anumang landas sa pagsusuri nito" (sariling salin). ${ }^{2}$

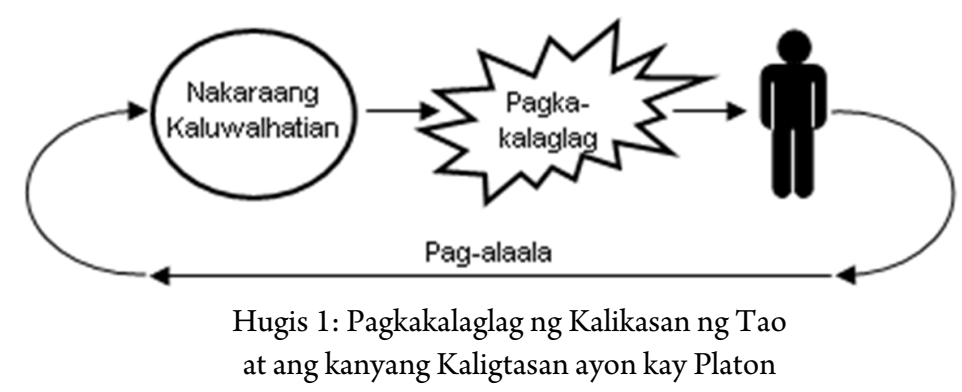

Si Kierkegaard ay may kahalintulad na modelo ng pag-iiral ng buhay na tao sa loob ng panahon na mahalaga rin para sa proyekto ni Heidegger. Bilang isang Kristiyanong pantas, tahasang naniniwala si Kierkegaard na may nalaglag na kalikasan (fallen nature) ang tao. Simboliko itong isinalaysay sa paglabag nina Adan at Eba sa utos ng Dios, at matutunghayan sa kahinaang loob ng tao kaharap ang nakapaligid na mga tukso. Para sa kanya matatamo ang kaligtasan ng isang Kristiyano sa paulit-ulit niyang pagpapahayag ng kanyang pananampalataya habang naglalakbay patungo sa inaasahang kaluwalhatian (tingnan ang hugis 2). Kung pabalik sa nakaraan ang kaligtasan para kay Platon, papunta naman ito sa hinarap para kay Kierkegaard.

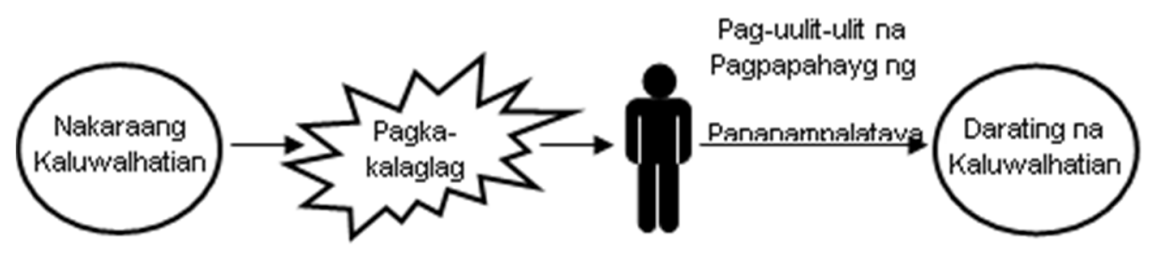

Hugis 2: Pagkakalaglag ng Kalikasan ng Tao at ang kanyang Kaligtasan ayon kay Kierkegaard

Pinagsama-sama ni Heidegger ang dalawang magkakasalungat na modelo nina Platon at Kierkegaark para masagot niya ang kanyang sariling tanong kung ano ang kahulugan ng pag-iiral (Being) ng buhay ng tao (Dasein). Bilang isang sekular na pilosopo, pinalitan ni Heidegger ang mga relihiyosong elemento ng dalawang pantas. Sa halip na banggitin niya ang pinagmumulang kaluwalhatian,

${ }^{2}$ Ibid., 39.

(C) 2012 F.P.A. Demeterio

http://www.kritike.org/journal/issue 11/demeterio june2012.pdf ISSN 1908-7330 
tinalakay niya ang orihinal at malalim na pakay ng tao, na ayon sa kanya ay ang pagpapahalaga sa kinabukasan; at sa halip na banggitin ang inaasahang kaluwalhatian, tinalakay niya ang minimithi ng taong matatamo niya ang kanyang mga potensyal pagdating ng araw (tingnan ang hugis 3 ).

May kanya-kanyang magagandang pangarap para sa sariling buhay ang bawat isa sa atin. Ngunit mas marami sa atin ang nabibigong makamtan ang mga gunitaing ito dahil madalas nakakalimutan natin ang mga ito sa gitna ng ating pagiging abala at pag-aatupag sa mga mabababaw na problema at aliw na tumatambad sa ating pang-araw-araw na buhay. Ang isang mag-aaral, halimbawa, na may maningning na mithiing maging isang dakilang doktor, o abogado, o arkitekto, noong una siyang sumabak sa kolehiyo, ay madaling malilihis sa kanyang hangarin dahil sa samut-saring problema, kaligayahan, pagsubok, at iba pang mga bagay na kaliwat-kanan ay hahatak sa kanya palayo sa anumang naangkop na landas. Isang araw gigising na lamang siya na wala na sa kanyang kalooban ang orihinal na mithiing unang nagdadala sa kanya sa kolehiyo. Ito ang ibig sabihin ni Heidegger kung papaano kayang hatakin ng kasalukuyan ang tao palayo sa kanyang orihinal at malalim na pakay sa buhay. Para kay Heidegger, may nalaglag na kalikasan (fallen nature) ang tao, dahil nalaglag siya palayo sa kanyang pakay at mithiin sa buhay.

Kapag hindi na malinaw ang nakaraan at kinabukasan ng tao magmimistula siyang isang patay na sanga na inaanod na lamang ng agos ng ilog. Magkakaroon siya ng isang buhay na walang direksyon at kahulugan, sa halip umaapaw sa kaba, pagkakadismaya, at pagkakabalisa. Kalaunan mawawalan na siya ng kanyang indibidwalidad na malulusaw kahalo ang masang kinamumuhian ng Alemang pilosopo na si Friedrich Nietzsche (1844-1900). Sa larangan ng kilos at galaw, matutunghayan ang sintoma ng pagkakalaglag ng kalikasan ng tao sa kanyang paghahanap ng mga pinakamadadaling lusot at remedyo; at sa larangan ng kaalaman, sa kanyang basta na lamang pagtatanggap kung ano ang sinasabi ng tradisyon at mas nakararami.

Para kay Heidegger, ang kaligtasan ng tao ay matatamo lamang sa kanyang pagbabalik muli at pag-alaala sa kanyang malalim at orihinal na pakay (tingnan ang hugis 3). Mangyayari lamang ito kapag hinaharap niya nang tapatan ang katotohanang may tiyak na kamatayang naghihintay sa dulo ng kanyang buhay. Tiyak at personal ang kamatayan. Walang sinuman ang maaaring magsabi na huwag itong mangyari sa kanya, at walang sinuman ang maaaring makiusap sa ibang tao na siya na lamang ang aako sa kanyang kamatayan. Ang kanyang sariling, tiyak at personal na kamatayan, kapag kanyang hinaharap ito ng tapatan ay ang magpapaalaala sa tao na ang buhay ay may hangganan at kung gusto niyang matatamo pa ang kanyang mga minimithi sa buhay ay dapat kumilos na siya.

Banggitin natin bilang halimbawa ang pilosopong si Kierkegaard, na napapaniwala ng kanyang sariling ama, na siya at ang kanyang lahat na mga kapatid 
ay mamamatay bago sila dadating sa kani-kanilang ika-34 na kaarawan (ang edad ni Hesu Kristo noong siya ay ipinako sa krus) dahil daw sa isang kasalanang nagawa ng kanyang ama. Dahil naniniwala siya na maikli lamang ang kanyang magiging buhay pinilit niyang naisulat ang kanyang napakaraming libro at sa proseso ay naging isang dakilang pilosopo, kritiko at dalubhasa sa teolohiya, kahit pa man napakabata nga naman talaga siyang pumanaw sa edad na 42. Ang pagmuni-muni ng tao sa kanyang sariling kamatayan ay magtutulak sa kanya na bawiin muli ang nawala niyang indibidwalidad, alalahanin muli ang kanyang mga mithiin at pakay sa buhay at kumilos papunta sa kanyang pinapangarap na kinabukasan (tingnan ang hugis 3 ).

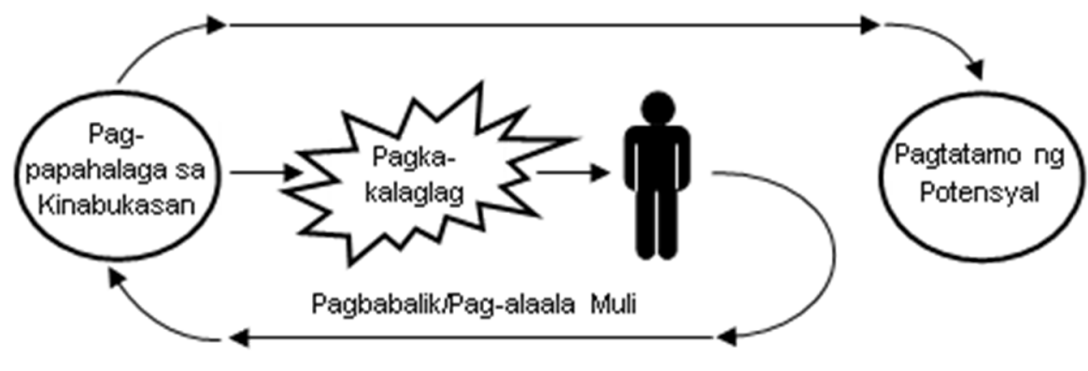

Hugis 3: Pagkakalaglag ng Kalikasan ng Tao

at ang kanyang Kaligtasan ayon kay Heidegger

Kapag ang kaligtasan ay paurong para kay Platon, at pasulong para kay Kierkegaard, ito ay sirkular na galaw para kay Heidegger. Naniniwala si Heidegger na ang kaligtasan ng tao ay isang pagbabalik at pagkukuha muli sa nakalimutan niyang pakay sa buhay, at susundan ito ng masigasig na kilos patungo sa pagsasakatuparan ng mga pakay na ito (tingnan ang hugis 3 ).

\section{Pag-uunawa at Proseso ng Interpretasyon ayon Kay Heidegger}

Dahil sirkular ang istraktura ng pag-iiral (Being) ng buhay ng tao (Dasein), naniniwala si Heidegger na ang istraktura ng kanyang pag-uunawa ay sirkular din. Ipinaliwanag ni John Caputo, isa sa mga ekspersto sa pilosopiya ni Heidegger: "binigyan ni Heidegger ang katagang 'sirkulo ng hermenyutika'—na dati ay may epistemolohikal at metodikal na mga kahulugan lamang-ng ontolohikal na bigat. Umuunawa tayo kagaya ng paguunawa natin dahil ganito tayo umiiral (Being). Ang pag-uunawa ay sumusunod sa paraaan ng pag-iiral (Being): intelligere sequitor esse" (sariling salin). ${ }^{3}$

3 John Caputo, Radical Hermeneutics: Repetition, Deconstruction, and the Hermeneutic Project (Bloomington: Indiana University Press, 1987), 61.

(c) 2012 F.P.A. Demeterio

http://www.kritike.org/journal/issue 11/demeterio june2012.pdf ISSN 1908-7330 
Sa pananaw ng isang taong nahuhulog palayo sa kanyang makatotohanang kalikasan, ang mga konsepto sa kanyang paligid ay nagmimistulang nahuhulog din palayo sa kani-kanilang mga makatotohanang kahulugan. Nabanggit na natin na ilan sa mga sintoma sa pagkakalaglag ng kalikasan ng tao ay ang kanyang paghahanap ng mga pinakamadadaling lusot at remedyo, at basta na lamang pagtatanggap kung ano ang sinasabi ng tradisyon at mas nakararami, gaano man kababaw ito. Katumbas ng nalaglag na kalikasan (fallen nature) ng tao ay ang mga bagay na nagmimistulang nalaglag din, sa puntong nababalotan ang mga ito ng hindi makatotohanang kahulugan. "Kapag ang tradisyon ay siyang naghahari," ipinaliwanag ni Heidegger, "kikilos ito sa paraan na anuman ang kanyang ipapahayag ay hindi na makukuhaan ng tunay kahulugan. .. at sa halip ay magiging nakakubli” (sariling salin). ${ }^{4}$

Ayon kay Heidegger may maraming paraan ng pagkakakubli ng kahulugan. Una, maaaring nakakubli ang kahulugan dahil hindi pa ito nadiskubre ng tao. ${ }^{5}$ Pangalawa, maaaring nadiskubre na ang isang kahulugan at natabunan muli nang lubusan sa puntong halos wala na itong ipinagkakaiba sa unang paraan ng pagkakakubli. Pangatlo, maaaring nadiskubre na rin ang isang kahulugan at natabunan muli ng samu't saring kahulugan sa puntong hindi na natin makikilala ang orihinal na kahulugan. ${ }^{6} \quad$ Mapagkunwari ang kahulugang nagkakubli sa paraang ito. Sabi ni Heidegger: "Ang pagkukubling ito bilang pagkukunwari ay ang mas madalas mangyayari at ang mas mapanganib dahil ang tsansa na ito ay makakalinlang at magtutulak sa landas ng maling pagkakaunawa ay mas malaki at mas mahirap labanan" (sariling salin). ${ }^{7}$

Hango sa mga sinaunang Griyego ang depinisyon ng katotohanan para kay Heidegger: ang aletheia, o ang pagtatanggal ng belo (unveiling) ng isang konsepto kasunod ng pagpapahayag nito ng kanyang kahulugan. Ngunit hindi ito kusang mangyayari kapag napabalutan ang konsepto ng samu't saring kahulugan. Kaya marahas ang unang hakbang ng proseso ng hermenyutika, dahil babaklasin nito ang mga nakakapit na kahulugan na silang nagbabaon sa orihinal na kahulugan ng isang konsepto. Kapag nalinis na ang konsepto, may kakayahan na itong magtanggal ng kanyang belo at magpahayag ng kanyang tunay na kahulugan.

Sa puntong ito walang pinagkakaiba ang hermenyutika ni Heidegger sa penomenolohiya na itinuturo ng kanyang gurong si Edmund Husserl (18551938). Dahil para kay Husserl ang tao ay dapat tatabi lamang sa bagay o konseptong kanyang pinag-aaralan, na walang dalang anumang asumsyon, prekonsepsyon o bias, at pasensyosong hayaan lamang ang bagay o konsepto na

\footnotetext{
${ }^{4}$ Heidegger, Being and Time, 42.

5 Tingnan sa Ibid., 60.

${ }^{6}$ Tingnan sa Ibid., 60.

${ }^{7}$ Ibid., 60.
} 
magpapahayag sa kanya ng kahulugan nito. ${ }^{8}$ Matapos baklasin ni Heidegger ang nakakapit na mga hindi makatotohanang kahulugan nandoon na siya sa kinaroroonan ni Husserl na katabi ang konsepto o bagay na handa nang magtanggal ng kanyang belo at magpapahayag ng kanyang nakatagong kahulugan.

Ngunit sa halip ng isang pasensyosong paghihintay sa tabi ng sinusuring bagay o konsepto na walang anumang dala-dalang asumsyon, prekonsepsyon at bias, kagaya sa penomenolohikal na pamamaraan ni Husserl, lumihis si Heidegger sa landas na ginawa ng kanyang guro at kinuwestiyon niya ang posibilidad mismo na kaya nga ng tao na lubusang isantabi ang kanyang mga asumsyon, prekonsepsyon at bias. Hindi naniniwala si Heidegger na kaya itong gawin ng sinumang tao. Kaya sa halip na isantabi ang mga ito iminungkahi ni Heidegger na ang mga asumsyon, prekonsepsyon at bias ng tao ay silang gawing mga instrumento sa pag-uunawa.

Dito lumalabas ang nabanggit na nating sirkular na istraktura ng paguunawa, na kahalintulad sa pangingisdang gamit ang lambat, at kung saan ang mga bagay o konsepto ay silang mga isda at ang mga pasiunang-balangkas ng paguunawa (forestructures of understanding) ay silang tumatayong lambat. Ang mga pasiunang-balangkas ng pag-uunawa ay ang mga asumsyon, prekonsepsyon at bias na nandiyan na sa kamalayan ng tao bago pa man siya haharap sa sinusuri niyang bagay at konsepto. Sabi nga ni Heidegger: "Ang pagsusuri, bilang isang paghahanap, ay dapat nagagabayan, bago pa man ito mag-uumpisa, ng konsepto ng kung ano ang hinahanap" (sariling salin). ${ }^{9}$ Kagaya ng paghagis ng mangingisda ng kanyang lambat para makahuli ng isda, dapat ding ihagis ng tao ang kanyang mga pasiunang-balangkas ng pag-uunawa para makuha niya ang kahulugan ng bagay o konseptong kanyang sinusuri. Ang mga proseso ng paghahagis (kilos palayo sa tao) at paghahatak (kilos papunta sa tao) ang silang gumuguhit ng isang sirkular na istraktura ng pag-uunawa (tingnan ang hugis 4).

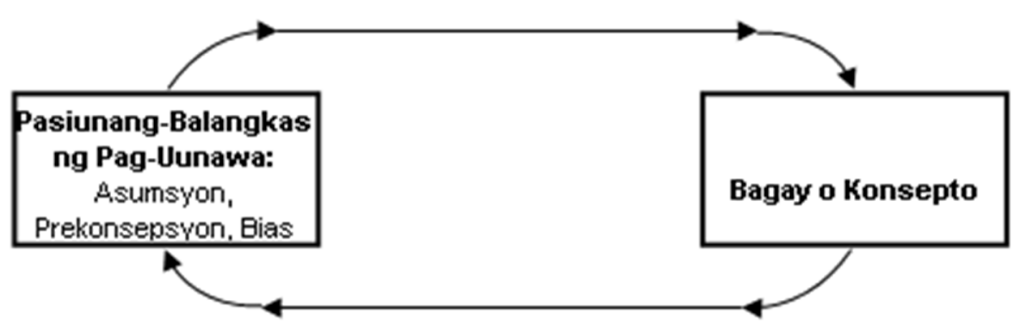

Hugis 4: Ang Sirkular na Istraktura ng Pag-Uunawa

8 Tingnan sa Christian Beyer, "Edmund Husserl," sa Stanford Encyclopedia of Philosophy,

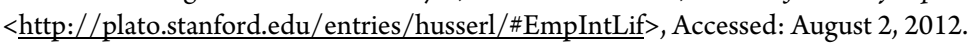

${ }^{9}$ Heidegger, Being and Time, 25.

(c) 2012 F.P.A. Demeterio

http://www.kritike.org/journal/issue 11/demeterio june2012.pdf

ISSN 1908-7330 
Kung para kay Friedrich Schleiermacher (1768-1834) tumutukoy ang sirkulo ng hermenyutika sa sirkular na ugnayan ng teksto at ng konteksto (dahil maiintindihan lamang natin ang teksto sa pamamagitan ng konteksto, at ang konteksto sa pamamagitan ng teksto) at ng partikular na bahagi ng teksto at ng kabuoan nito (dahil maiintindihan lamang natin ang partikular sa pamamagitan ng kabuoan, at ang kabuoan sa pamamagitan ng particular), para kay Heidegger tumutukoy ito ngayon sa sirkular na ugnayan ng tao at ng konsepto, bagay, o tekstong kanyang sinusuri (tingnan ang hugis 4 ). ${ }^{10}$

Para kay Heidegger mayroong tatlong uri ng pasiunang-balangkas ng paguunawa (forestructures of understanding): ang pasiunang-pag-angkin, o fore-having; ang pasiunang-pagmasid, o fore-sight; at ang pasiunang-paghimay, o foregrasping. ${ }^{11}$ Ang pasiunang-pag-angkin ay ang pagkakaroon ng tao sa kanyang kamalayan ng isang malawakang larawan sa konteksto na kinabibilangan ng bagay o konseptong kanyang susuriin pa lamang (tingnan ang hugis 5).

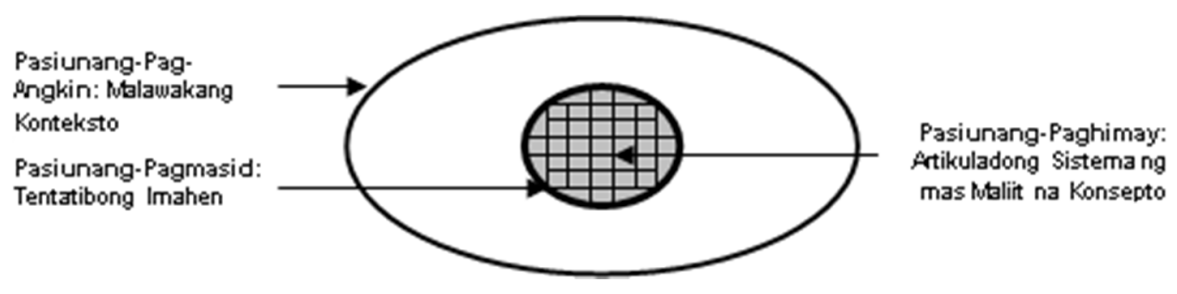

Hugis 5: Ang Tatlong Pasiunang-Balangkas ng Pag-Uunawa

Ang pasiunang-pagmasid naman ay ang pagkakaroon ng tao sa kanyang kamalayan ng isang tentatibo at malabong imahen ng bagay o konseptong kanyang susuriin pa lamang (tingnan ang hugis 5). Ang pagkakaiba ng pasiunang-pagangkin at pasiunang-pagmasid ay makikita sa lawak ng kani-kanilang pokus. Ang pasiunang-pag-angkin ay nakatuon sa konteksto, habang ang pasiunang-pagmasid ay nakatuon sa mismong bagay o konseptong susuriin.

Ang pasiunang-paghimay naman ay ang pagkakaroon ng tao sa kanyang kamalayan ng isang artikuladong sistema ng mga mas maliliit na konsepto para huliin ang detalye ng bagay o konseptong susuriin pa lamang (tingnan ang hugis 5). Ang pagkakaiba ng pasiunang-pagmasid at pasiunang-paghimay ay makikita pa rin sa lawak ng kani-kanilang pokus. Ang pasiunang-pagmasid ay nakatuon sa kabuan ng bagay o konseptong susuriin, habang ang pasiunang-paghimay ay nakatuon sa kung ano ang nilalaman ng bagay o konseptong ito.

10 Tingnan sa Feorillo Demeterio, “Ang Hermenyutika nina Schleiermacher at Dilthey bilang Batayang Teoretikal sa Araling Pilipino,” sa Kritike, 5:1 (June 2011), 2-7.

${ }^{11}$ Tingnan sa Heidegger, Being and Time, 191.

(c) 2012 F.P.A. Demeterio

http://www.kritike.org/journal/issue 11/demeterio june2012.pdf

ISSN 1908-7330 
Ang mga pasiunang-balangkas ng pag-uunawang ito ay silang inihahagis ng tao sa bagay o konseptong kanyang sinusuri. Malabo, wala masyadong laman, mapurol, at tentatibo lamang ang mga pasiunang-balangkas na ito. Pero matapos nilang paikotan ang bagay o konseptong sinusuri, sila ay lumilinaw, nagkakaroon ng laman, tumatalas at nagiging mas ganap. Kaya kapag wala ang bagay o konsepto na sinusuri wala ring masyadong saysay ang mga pasiunang-balangkas ng pag-uunawa, at kapag wala naman ang mga pasiunang-balangkas ng pag-uunawa hinding hindi rin mauunawaan ng tama ng tao ang anumang bagay o konsepto.

Para kay Heidegger ang pag-uunawa, na isang pragmatiko, eksistensyal at hindi metodikong gawain, ay siyang ugat $\mathrm{ng}$ mas teknikal na proseso ng interpretasyon. Sila ay magkakaiba lamang sa antas ng kaganapan, sa katuwirang ang proseso ng interpretasyon ay ang pinakaganap na anyo ng pag-uunawa. Dahil sa kwalitatibong pananaw pareho ang pag-uunawa at proseso ng interpretasyon, pareho din silang nag-uumpisa gamit ang tatlong uri ng pasiunang-balangkas ng pag-uunawa. Ang pinakakrusyal na pagkakaiba ng dalawang mental na prosesong ito ay ang reyalisasyon na ang proseso ng interpretasyon ay nangangailangan ng sadyang pagpapatalas at pagpapayaman ng mga pasiunang-balangkas ng paguunawa. Ibig sabihin, kahit sino sa atin ang may kakayahang umunawa, ngunit tanging mga tao lamang na may sapat na paghahanda ang maaaring sumabak sa landas ng interpretasyon o hermenyutika.

Isa pang mahalagang pagkakaiba ng pag-uunawa at $\mathrm{ng}$ proseso $\mathrm{ng}$ interpretasyon ay ang pangangailangan ng pagbabaklas ng mga nakakapit na maling kahulugan. Hindi pangkaraniwang kinakailangan ang gawaing ito sa pangaraw-araw nating pag-uunawa, kahit pa man dapat din tayong maging mulat na ang mga kaalamang tinatanggap natin mula sa tradisyon at nakararaming kapwa natin ay maaaring magdudulot lamang ng maling pag-uunawa. Ngunit para sa proseso ng interpretasyon o hermenyutika, ang pagbabaklas sa mga nakakapit na maling kahulugan ay kailangang isasagawa muna bago pa man gamitin ang mga pasiunang-balangkas ng pag-uunawa.

Dialohikal ang tawag natin sa hermenyutika ni Heidegger dahil nagmimistulang isang dialogo ang ugnayan $\mathrm{ng}$ tao at ng konsepto, bagay o tekstong kanyang sinusuri. Kahit na sa kaisipan ni Heidegger ang ganitong istraktura ng ugnayan ay hindi pa masyadong matingkad, lilinaw ang anyo nito habang pinapatalas ito ng mga sumusunod na pantas na naimpluwensyahan ng kanyang hermenyutika, sina Bultmann at Gadamer.

\section{Kritisismo ng Porma Bilang Biblikal na Hermenyutika ayon kay Bultmann}

Si Rudolf Bultmann (1884-1976), isang Alemang dalubhasa sa teolohiya at sa pag-aaral ng bibliya, ay naging katrabaho ni Heidegger doon sa Universidad 
ng Marburg noong mga taong 1922 hanggang 1928. Kahit pa man mas matanda si Bultmann kay Heidegger ng limang taon, at kahit pa man nagkakahiwalay ang kanilang mga landas mula noong dekada 30 dahil naging kasapi si Heidegger sa partidong Nazi ni Hitler habang naging kritiko naman si Bultmann sa mga kalabisan ng kilusang ito, malinaw na naimpluwensyahan si Bultmann ng pilosopiya ni Heidegger, sa puntong dinala niya ang dialohikal na hermenyutika sa larangan ng teolohiya at pag-aaral ng bibliya.

Ngunit bago pa man nakasama ni Bultmann si Heidegger, kilala na siya bilang isang pangunahing dalubhasa sa biblikal na pag-aaral ng bagong tipan. Kasama niya ang isa ring Alemang dalubhasa sa teolohiya at bibliya na si Martin Dibelius (1883-1947), binuo nila ang kritisismo ng porma (form criticism) para sa unang tatlong libro ng bagong tipan, ang mga ebanghelyo nina Mateo, Marko at Lukas. Tinaguriang mga 'sinoptikong ebanghelyo' ang tatlong librong ito, isang pangalang hango sa mga Griyegong salitang ugat na "syn" at "optiko" na nangangahulugang pinagsamang tingin, dahil ang nilalaman nila umano ay maaaring ihanay na magkakatabi at basahin bilang iisang salaysay.

Isang espesyal na uri ng gramatikal na rekonstraksyon ang kritisismo ng porma na binuo nina Bultmann at Dibelius. Ayon sa metodong ito ang tatlong sinoptikong ebanghelyo ay hindi daw mga historikal na sanaysay na isinulat ng mga taong aktwal na nakasaksi sa buhay ni Hesu Kristo. Sa halip, binuo lamang daw ang mga ito ng mga may-akdang nabubuhay ilang henerasyon matapos ang pagkamatay ni Hesu Kristo mula sa ibat-ibang nakasulat at salimbibig na mga materyal na pinag-iingatan at pinapahalagahan ng mga sinaunang simbahan.

Para kay Bultmann nahahati sa dalawang grupo ang mga materyal at batis na ito: ang mga diskurso ni Hesus at ang mga naratibong materyal (tingnan ang hugis 6). Nahahati naman ang mga diskurso ni Hesus sa dalawa ring grupo: ang mga apophthegm at mga kasabihan ng panginoon (tingnan ang hugis 6). Hinango ni Bultmann ang salitang "apophthegm" mula sa pag-aaral ng Griyegong panitikan. Ipinaliwanag niya: "ang tiyak na katangian ng isang apophthegm ay ang kanyang pagsasalarawan ng isang hindi mahalagang eksena na nagbibigay ng balangkas para sa isang importanteng salita ng bayani, pilosopo, relihiyosong lider, at ibang katulad na tauhan. Ang importanteng bagay dito ay ang salita mismo; ang naratibong balangkas ay nagsisilbing isang sitwasyon lamang na lulunsaran ng salita." 12 Ang mga apophthegm, mga kasabihan ng panginoon, at mga naratibong materyal ay nahahati sa sumusunod na mga grupo:

12 RudolfBultmann, "The New Approach to the Synoptic Problem," sa Ogden, Schubert, Existence and Faith: Shorter Writings by Rudolf Bultmann (London: Fontana Library, 1964), 52.

(C) 2012 F.P.A. Demeterio

http://www.kritike.org/journal/issue 11/demeterio june2012.pdf

ISSN 1908-7330 


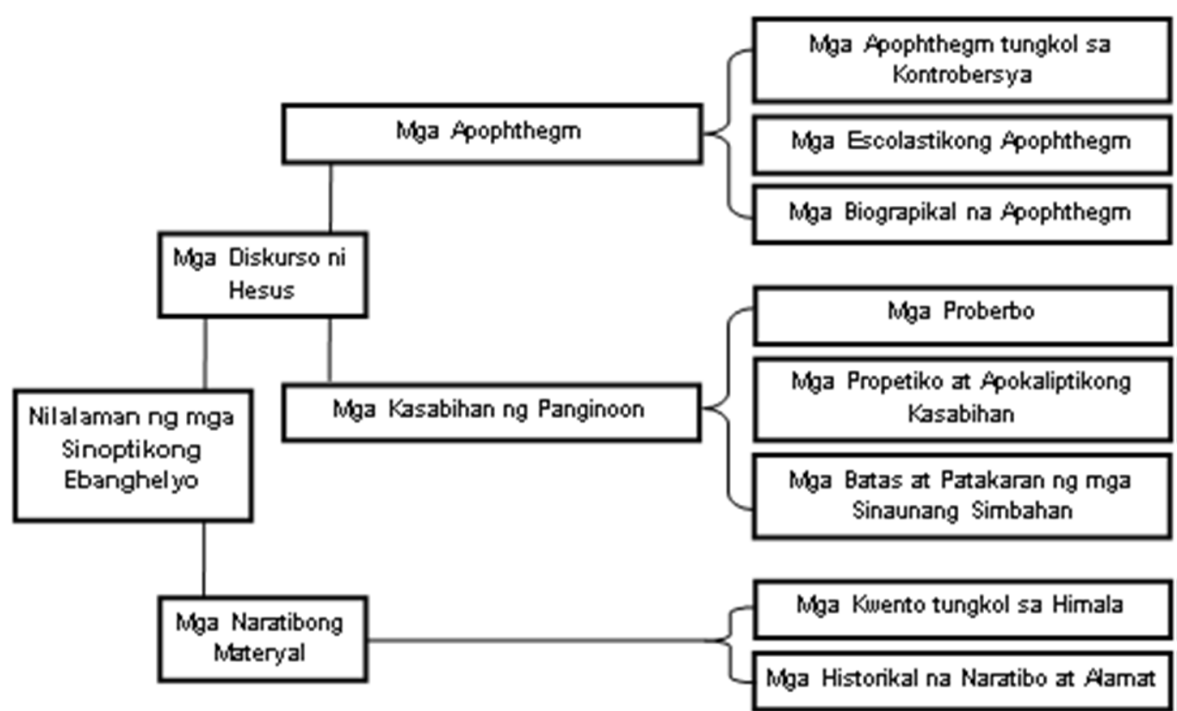

Hugis 6: Ang mga Materyal na Nilalaman ng mga Sinoptikong Ebanghelyo

Sa mas madaling salita ang tatlong sinoptikong ebanghelyo ay isinulat ng kani-kanilang may-akda gamit ang hindi kukulang sa walong uring materyal at batis: 1) ang apophthegm tungkol sa kontrobersya, 2) ang mga eskolastikong apophthegm, 3) ang mga biograpikal na apophthegm, 4) ang mga proberbo, 5) ang mga propetiko at apokaliptikong kasabihan, 6) ang mga batas at patakaran ng mga sinaunang simbahan, 7) ang mga kwento tungkol sa himala, at 8) ang mga historikal na naratibo at alamat. May kanya-kanyang pinanggagalingan, katangian at istraktura, ang bawat uri ng materyal at batis na ito. Kaya malinaw na hindi dapat ituturing na historikal ang nabuong ebanghelyo nina Mateo, Marko at Lukas dahil may ibat-ibang katayuan ang mga elementong nakapaloob sa bawat ebanghelyong ito.

Nagmumungkahi ang kritisismo sa porma ni Bultmann na alamin muna ng mambabasa kung ano ang partikular na pinanggagalingang materyal o batis ng isang sinoptikong teksto para malaman niya kung paano ituturing ang kanyang tekstong sinusuri. Kagaya ng dapat babasahin lamang natin ang isang nobela bilang isang nobela, o ang isang tula bilang isang tula, dapat babasahin din natin ang isang apophthegm bilang isang apophthegm, o ang isang kwentong himala bilang isang kwentong himala. Ang pinakamensahe ng kritisismo sa porma nina Bultmann at Dibelius ay ang direktiba na alamin muna ang partikular at espisipikong porma ng teksto bago pa man gumawa ng anumang interpretasyon. Sa oras na nagkakamali ang isang mambabasa sa pagkikilala sa porma ng isang teksto, magiging kwestiyonable ang kanyang magagawang interpretasyon. 


\section{Eksistensyalistang Hermenyutika ayon kay Bultmann}

Nang makasalamuha ni Bultmann si Heidegger noong mga taong 1922 hanggang 1928 doon sa Universidad ng Marburg, unti-unti niyang binuo ang isang eksistensyalistang hermenyutika na nakabatay sa teorya ni Heidegger tungkol sa proseso ng pag-uunawa ng tao. Sinunod ni Bultmann ang paniniwala ni Heidegger na hindi kaya ninuman ang lumapit sa anumang teksto na walang dala-dalang asumsyon, prekonsepsyon at bias sa kanyang kamalayan para maisagawa ang isang malinis na interpretasyon. Sinunod din ni Bultmann ang mungkahi ni Heidegger na sa halip na itakwil ang mga mental na bagaheng ito, gagawin na lamang silang pundasyon ng pag-uunawa at proseso ng interpretasyon. Kaya kung merong mga pasiunang balangkas ng pag-uunawa (forestructures of understanding) si Heidegger, pinapahalagahan naman ni Bultmann ang kanyang tinatawag na pasiunang paguunawa (pre-understanding).

Para kay Bultmann ang pasiunang pag-uunawa (pre-understanding) ay tumutukoy sa mga katanungan at paksang pinagkaka-abalahan ng mambabasa na taglay niya sa kanyang kamalayan bago pa man siya lumapit sa tekstong kanyang susuriin. Sinabi ni Bultmann: "walang interpretasyon (exegesis) ang magaganap na walang presuposisyon dahil hindi tabula rasa ang mambabasa (exegete), sa halip lumalapit siya sa teksto na may dala-dalang espisipikong mga katanungan o hindi kaya mga espisipikong paraan sa pagtatanong, at kaya mayroon na siyang ideya kahit paano tungkol sa paksa na nag-anyaya sa kanya na suriin ang teksto." ${ }^{13}$

Ang dala-dalang mga katanungan, paraan ng pagtatanong, at iba pang mga prekonsepsyon ng mambabasa ay natural lamang na makakaapekto sa kalalabasan ng anumang isasagawang interpretasyon. Dahil dito tanggap ni Bultmann na maaaring magkakaroon ng ibat-ibang interpretasyon ang iisang teksto. Halimbawa ang mga likha ng pintor na si Ang Kiukok ay maaaring susuriin ng isang Marxista, o ng isang Sikoanalista, o ng isang pormalista, o ng isang dalubhasa sa kasaysayan ng sining, at ng kung sino pa man. Dahil magkakaiba-iba ang pinagmumulang pananaw at ang mga pinagkakaabalahan ng mga kritikong ito, hindi makapagtataka na magkakaiba-iba rin ang maisusulat nilang nilang mga kritisismo kahit pa man parehong koleksyon ng mga obra ni Ang Kiukok ang kanilang sinuri. Dadag pa dito, ang mga pinagmumulang perspektibo ng mga kritiko ay maaari ding dumami pa sa paglipas ng panahon dahil magkakaiba-iba rin ang mga katanungan at pinagkakaabalahan ng bawat yugto ng kasaysayan. Kaya kahit ang interpretasyon ng isang Marxista, o ng isang pormalista noong dekada sitenta, ay maaaring maiiba sa interpretasyon ng isang kasalukuyang Marxista, o ng isang kasalukuyang pormalista.

${ }^{13}$ Rudolf Bultmann, “Is Exegesis without Presupposition Possible," sa Ogden, Schubert, Existence and Faith: Shorter Writings by Rudolf Bultmann, 342. 
Kapag wala nang hangganan ang bilang ng posibleng maging interpretasyon ng isang teksto, nangangahulugan ba ito na Para kay Bultmann lubusan nang naging malaya ang mambabasa na gumawa ng kahit anong interpretasyon na gusto niya? Wala na bang mga prinsipyong maaaring gumabay sa mga mambabasa? Naniniwala si Bultmann na hindi lubusang malaya ang mambabasa na gumawa ng kahit ano na lamang na interpretasyon, at na may mga prinsipyo pa rin na dapat gagabay sa proseso ng interpretasyon at hermenyutika. Una, dapat batid ng mambabasa na ang kanyang pasiunang pag-uunawa ay inisyal na balangkas lamang, at isa lamang sa napakaraming inisyal na balangkas.

Pangalawa, ang pasiunang pag-uunawa ay dapat payamanin at payabongin sa pamamagitan ng aktwal na pakikisalamuha sa tekstong sinusuri. Dapat masinsinan at sistematiko pa rin ang proseso ng interpretasyon. Isinulat ni Bultmann: "ang bawat interpretasyon (exegesis) na ginabayan lamang ng mga dogmatikong prekonsepsyon ay hindi nakikinig sa sinasabi ng teksto, at sa halip ay nagpupumipilit na sabihin lamang ng teksto ang mga bagay na gusto niyang marinig." 14 Hindi saradong paninindigan ang pasiunang pag-uunawa, at bukas ito sa posibilidad na taliwas ang kanyang mga asumsyon, prekonsepsyon at bias sa lalabas na interpretasyon. Hindi rin isinantabi ni Bultmann ang kanyang mabusising kritisismo sa porma, at kung kailangan itong gamitin sa anumang proyekto ng interpretasyon, dapat lamang itong gamitin.

Pangatlo, iiwasan dapat ng mambabasa na isipin na ang kanyang pinagmumulang pananaw ay siya lang dapat ang nag-iisang tamang pananaw, o hindi kaya ang pinakamahusay at pinakamabisang pananaw. Binigyang diin ni Bultmann: "ang historikal na larawan ay nagiging mali lamang kapag ang isang paraan sa pagtatanung ay ituturing na nag-iisang tamang paraan ng pagtatanonghalimbawa, kapag ang lahat ng kasaysayan ay ikinahon bilang pang-ekonomikong kasaysayan." ${ }^{15}$

Bukod sa kanyang pagbatay sa kanyang hermenyutika sa eksistensyalistang pagdalumat sa istraktura ng pag-uunawa ni Heidegger, pinalalim pa ni Bultmann ang eksistensyalismo sa kanyang hermenyutika sa pamamagitan ng pagteteorya na ang ilang mga piling teksto, partikular na iyong mga nanggagaling sa larangan ng pilosopiya, relihiyon at literatura, ay maaaring may mga nakatagong mensahe na makatutulong sa atin sa pagsasagot sa eksistensyal na katanungan tungkol sa saysay ng ating buhay. Kung ating maalaala sentral ang tanong na ito sa pilosopiya ni Heidegger, at penomenolohikal at dedaktibo niyang sinagot kung ano ang ibig sabihin sa pag-iiral ng buhay ng tao (Dasein). Para kay Bultmann, hermenyutikal at indaktibong masasagot ang parehong katanungan sa pamamagitan ng ilang mga piling teksto mula sa mga larangan ng pilosopiya,

${ }^{14}$ Ibid., 343.

${ }^{15}$ Ibid., 346. 
relihiyon at literatura. Batay ito sa katotohanang ang tao, kahit saang lupalop at anong panahon siya nabubuhay, ay binabagabag ng isang pundamental na katanungan kung ano ang saysay ng pag-iiral ng kanyang buhay. Kaya sa mga teksto ng kanyang pilosopiya, relihiyon, at literatura lalabas at lalabas ang kanyang mga munimuni at kasagotan sa eksistensyalistang problemang ito.

\section{Demitolohisasyon bilang Biblikal na Hermenyutika ayon kay Bultmann}

Matapos magkahiwalay ang kanilang landas ni Heidegger, pinalawig at pinalalim pa ni Bultmann ang kanyang hermenyutikang nakatuon sa biblikal na pag-aaral. Noong 1941, nabuo niya ang isang kontrobersyal na teorya at pamamaraan na tinatawag niyang "demitolohisasyon." Sa larangan ng biblikal na hermenyutika karugtong ito sa kanyang heneral na eksistensyalistang hermenyutika. Ibig sabihin, isang estratihiya ang demitolohisasyon para mas mapadali ang pagtukoy sa eksistensyalistang mensahe ng bibliya.

Kung sa mga teksto ng relihiyon, pilosopiya at literatura ay nakakubli ang isang eksistensyalistang mensahe, "kerygma" naman ang katumbas nito sa mga tekstong biblikal. Isang Griyegong salita ang "kerygma" na tumutukoy sa pinakamahalagang mensahe ng Dios na ipinahagag niya sa pagkatao ni Hesu Kristo. Kung sa heneral na eksistensyalistang hermenyutika nakatuon dapat ang mambabasa sa pag-uunawa kung ano ang sagot ng teksto sa tanong na kung ano ang saysay ng buhay ng tao, sa demitolohisasyon nakatuon dapat ang mambabasa sa pag-uunawa kung ano ang kerygma na taglay ng partikular na tekstong biblikal.

Ngunit, ayon kay Bultmann, ang kerygma ay hindi madaling matunghayan inihayag ito ng bibliya gamit ang mitolohikal na wika ng mga mayakda nito. Nakabatay ang wikang mitolohikal na ito sa kultura at panahon ng mga may-akda ng bibliya. Kaya ang mga taong nanggagaling sa ibang kultura at panahon ay maaaring mahihirapan sa pangtanggap sa mitolohiyang ginagamit ng mga may-akda bibliya. Ibig sabihin, sa halip na masisilbing daluyan ng kerygma ang wikang mitolohikal, maaari itong magiging balakid sa lubusang pag-uunawa ng bibliya. Halimbawa, ang ating kasalukuyang siyantipikong pananaw ay mahihirapang tumanggap sa kaliwat kanang mga himalang nanggyaari sa mga sanaysay ng bibliya. Batid ni Bultmann ang malaking posibilidad na ang mga modernong tao ay madidismaya lamang habang binabasa ang mga kwentong lumalabag sa kanyang kinatatayuang siyantipikong sukatan kung ano ang totoo at hindi. Sa halip na magpupursige ang modernong taong ito sa pagtukoy sa nakakubling kerygma, titigil na lamang ito sa pagbabasa at tuluyan nang isantabi ang buong bibliya.

Sa teorya at pamamaraan ng demitolohisasyon, itinuturo ni Bultmann na dapat kilatisin ng mambabasa ang tekstong biblikal para masasala niya kung alin sa 
mga elemento nito ang kerygmatiko at alin ang mitolohikal. Ang mga mitolohikal na elemento ay hindi kailangan burahin mula sa bibliya. Para kay Bultmann dapat lamang na ang mga elementong ito na naka-ugat sa wikang mitolohikal ng mga may-akda ng bibliya ay ituturing na hindi kasing halaga sa mga kerygmatikong elemento. Sa ganitong paraan, kahit anong kultura at panahaon ang pinanggagalinang ng isang mambabasa hindi na siya mahihirapang makipagsapalaran sa sapilitang pagtanggap ng mga mitolohikal na elemento ng bibliya at maaari na siyang dumeretso sa pag-uunawa sa pinakamahalagang elemento ng bibliya, na walang iba kung hindi ang kerygma.

Ang dialohikal na uri ng hermenyutika ay nakapaloob lamang sa eksistensyal na hermenyutika at teorya at pamamaraan ng demitolohisasyon ni Bultmann at hindi sa kanyang mas naunang kritisismo sa porma. Parehong nakabatay ang dalawang dialohikal na teorya at pamamaraan sa palitan ng mga mental na balangkas sa pagitan ng mambabasa at ng tekstong kanyang sinusuri. Ngunit ang istraktura ng dialogo ay hindi pa rin gaano katingkad kahit sa elaborasyon ni Bultmann sa hermenyutika ni Heidegger.

\section{Ang Prejudice bilang Pundasyon ng Hermenyutika ayon kay Gadamer}

Si Hans-Georg Gadamer (1900-2002), isang Alemang pilolohista at pilosopo, ay naging nakababatang kasamahan nina Heidegger at Bultmann doon sa Universidad ng Marburg. Gamit ang mga kaisipan ng mga Griyegong pilosopo na sina Platon at Aristoteles pinatingkad pa niya lalo ang istraktura ng dialogo sa hermenyutika na itinatag ni Heidegger.

Hindi katulad nina Heidegger at Bultmann na naging mga batikang dalubhasa sa pilosopiya at teolohiya habang bata pa lamang, sumikat si Gadamer noong siya ay saisentang taong gulang na at malapit na magretiro, matapos niyang mailathala ang kanyang pangunahing obra na Katotohanan at Pamamaraan (Truth and Method). Sa librong ito binatikos ni Gadamer ang pinakadambuhalang prejudice na dala dala ng Enlightenment. Ito ay walang iba kung hindi ang prejudice laban sa prejudice. Kinabitan ng Englightenment ang konsepto na dating nangangahulugan lamang na 'pasiunang paghuhusga' ng mga konotasyong hindi kanais-nais. Sa panahong mas tinatanggap na ng nakararami ang empirisismo ng siyensya, ang Cartesyanong pagdududa at ang sistematikong pagbubuo ng kaalaman, hindi lamang nawalan ng puwang at halaga ang prejudice, naging kabaligtaran pa ito ng mga mithiin ng Enlightenment.

Naniniwala si Gadamer na mahalaga ang prejudice, na nakapaloob sa kultura, tradisyon, at panlasa ng tao, sa kanyang pagkikilos sa harap ng anumang sitwasyon, sa kanyang pag-uunawa, at sa kanyang pagpapasya kung ano ang mabuti o masama, at maganda o hindi maganda. Lumalabas na isang hindi 
maitatakwil na batayan ang prejudice para sa mga praktikal na gawain ng isang komunidad. Ngunit sa pagkakahumaling ng Enlightenment sa mga bagay na siyantipiko, obhektibo at teoretikal, naisasantabi nito ang kultural, ang tradisyunal, at ang panlasa ng tao, dahil talaga namang nagkululang ang mga ito sa pagiging siyantipiko, sa pagiging obhektibo at sa pagiging teoretikal.

Sa larangan ng hermenyutika, matutunghayan natin ang pangungutya ng Enlightenment laban sa prejudice sa penomenolohiya ni Husserl, kung saan iminungkahi nito na ikahon na lamang ang mga prejudice para hindi sila makakasira sa mga proseso ng pag-uunawa at interpretasyon. Katulad ni Heidegger, sinuway din ni Gadamer ang penomenolohikal na patakaran ni Husserl. Imposible na mangyayari ang masinop na pagkakahon ng mga prejudice, dahil hindi naman alam ninuman kung alin sa mga nilalaman ng kanyang isipan ang mga prejudice at alin ang hindi, dahil maaaring iba sa kanyang mga prejudice ay may napakamalalim na na ugat sa puntong hindi na niya mamamalayan ang mga ito, at dahil kahit ikakahon man ang mga ito, patuloy silang nandoon sa kanyang isipan. Kaya, katulad din ni Heidegger, itinaguyod ni Gadamer na gamitin na lamang nang maayos ang mga prejudice sa proseso ng pag-uunawa at interptretasyon.

Aminado si Gadamer na hindi makatutulong sa isang tao ang lahat ng prejudice na nasa kanyang isipan. Dahil may mga bulag na prejudice na magsisilbing balakid sa proseso ng pag-uunawa at interpretasyon, o hindi kaya hahatak sa kanya patungo sa maling pag-uunawa at interpretasyon. Tanging ang mga lehitimong prejudice lamang ang mahalaga para kay Gadamer, dahil ang mga ito ang nagsisilbing daluyan ng pag-uunawa at interpretasyon. Subalit, kahit pa man malinaw para sa kanya na mayroong mga bulag at lehitimong prejudice, wala siyang malinaw at sistematikong pamantayan kung paano malalaman kung ang isang partikular na prejudice ay bulag ba o lehitimo. Naniniwala siya na walang siyantipiko, obhektibo at teoretikal na paraan sa pagsusuri kung ang isang prejudice ay bulag ba o lehitimo, dahil malalaman lamang daw kung anong uri ang isang prejudice matapos ang kongkreto, subhektibo, at praktikal na paggamit nito sa aktwal na proseso ng pag-uunawa at interpretasyon. Para kay Gadamer ang sirkulo ng hermenyutika na namana niya kay Heidegger ang siya ring proseso na maghihimay kung alin sa mga prejudice ang bulag at alin ang lehitimo.

\section{Ang Dialogo bilang Modelo ng Hermenyutika ayon kay Gadamer}

Gamit ang modelo ng dialogo mula sa sentral na kaisipan ni Platon pinatingkad ni Gadamer ang istraktura ng dialogo sa hermenyutika ni Heidegger. Nakita na natin na sa teorya ni Heidegger, hindi gaano ka linaw ang istrakturang ito, dahil hindi balanse ang mga katayuan ng tao at ng teksto. Mahirap isipin ang isang dialogo kapag tao at bagay ang magkakaharap, dahil pangkaraniwang 
nangyayari ang prosesong ito sa pagitan ng tao at isa pang tao. Naglahad ng inobasyon si Gadamer sa puntong ito sa pamamagitan ng pagbabalanse sa mga katayuan ng tao at teksto. Sa ganitong paraan magiging mas madaling isipin na mayroon ngang dialogo na maaaring magaganap sa pagitan ng mga ito.

Naipahiwatig na natin na pundasyunal ang prejudice sa hermenyutika ni Gadamer. Kaya kung mayroong prejudice ang mambabasa, mayroon ding prejudice dapat ang tekstong kanyang susuriin. Hindi naman mahirap tanggapin ito, dahil maaari naman talagang isipin na daladala ng isang teksto ang mga prejudice hindi lamang ng kanyang may-akda, kung hindi pati na ng kanyang pinagmumulang kultura at panahon.

Ngunit hindi nagtapos si Gadamer sa konsepto ng prejudice, sa halip tinipon niya ang mga ito sa ilalim ng isang mas malaking konseptong tinatawag niyang "abot-tanaw" (horizon). Isinulat niya: "unawain natin ang konsepto ng 'sitwasyon' sa pamamagitan ng pagbanggit na inirepresenta nito ang isang punto de bista na naglilimita sa posibilidad ng paningin. Kaya mahalagang parte ng konsepto ng sitwasyon ay ang konsepto ng 'abot-tanaw' (horizon). Ang 'abottanaw' (horizon) ay ang saklaw ng paningin na sumasakop sa lahat ng makikita mula sa partikular na punto de bista."16 Sa mas madaling salita, ang abot-tanaw (horizon) ay ang kalipunan ng mga kalaaman, damdamin at prejudice na taglay palagi ng isang tao sa kanyang kamalayan at nagsisilbing lunsaran ng kanyang paguunawa.

Panumbas ni Gadamer ang abot-tanaw (horizon) sa mga pasiunang balangkas ng pag-uunawa ni Heidegger. Para sa pagpapatingkad ng istraktura ng dialogo, may bentahe si Gadamer kay Heidegger, dahil mas madaling isipin na ang teksto ay may sariling 'abot-tanaw' (horizon) kaysa may sarili itong mga pasiunang balangkas ng pag-uunawa. Dagdag pa dito mas dinamiko ang konsepto ng 'abottanaw' (horizon) kaysa mga pasiunang balangkas ng pag-uunawa, dahil ang 'abottanaw' (horizon) ay bukas palagi sa pagbabago at rebisyon habang nakikisalamuha ito sa ibang mga 'abot-tanaw' (horizon). Sinabi ni Gadamer: "ang abot-tanaw (horizon) ay pawang isang bagay kung saan tayo gumagalaw at gumagalaw rin kasabay natin. Ang mga abot-tanaw (horizons) ay nagbabago para sa isang tao na gumagalaw." 17

Sa puntong ito isantabi muna natin ang masalimuot na modelo ng dialogo sa pagitan ng tao at teksto, at isipin muna natin ang istraktura ng karaniwang dialogo sa pagitan ng dalawang tao. Para kay Gadamer ang pakay ng dialogo ay ang pagsasanib ng mga abot-tanaw (fusion of horizons) ng dalawang taong ito, at ito ay matatamo naman sa pamamagitan ng kanilang pag-uusap at palitan ng mga kaalaman, damdamin, at prejudice. Kapag, halimbawa, may dalawang

16 Hans-Georg Gadamer, Truth and Method (New York: Continuum Publishing Company, 1999), 269.

${ }^{17}$ Ibid., 271. 
magkakaibigan na palaging magkakasama at nag-uusap, hindi tayo magtataka kung may malaking pagkakapareho ang kanilang mga pananaw sa maraming bagay dahil naiintindihan natin na sa nakaraang mahabang panahon nahubog at naimpluwensyahan na nila ang isat-isa. Ang paghuhubog at pag-impluwensya ng magkakaibigang ito sa isat-isa ay ang binabanggit ni Gadamer na proseso ng pagsasanib ng kanilang mga abot-tanaw (fusion of horizons).

Mahalagang makita natin na para matatamo ang pagsasanib ng mga abottanaw (fusion of horizons), mahaba, paulit-ulit at masinsinan dapat ang usapan at interaksyon ng dalawang tao (tingnan ang hugis 7). Dito natin matutunghayan na ang sirkulo ng komunikasyon, at ng hermenyutika, ay dapat pala paulit-ulit na umiikot hanggang matatamo ang pagsasanib ng mga abot-tanaw (fusion of horizons). Sa bawat ikot ng sirkulo nito napapalapit sa isat-isa ang dalawang abottanaw (horizon). Katulad ng isang pangkaraniwang dialogo, na hindi "isang tanong isang sagot" na pangyayari, ang sirkulo ng komunikasyon at hermenyutika ay mahaba-habang palitan ng mga pahayag, opinion, tanong, puna, paglilinaw, pagsasang-ayon, hindi pagsasaang-ayon, mungkahi at iba pa.

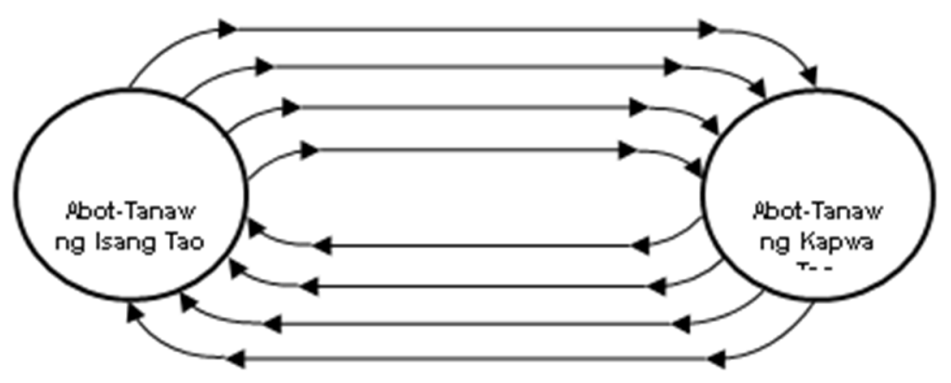

Hugis 7: Ang Paikot-ikot na Istraktura ng Dialogo

Matapos nating malaman na ang isang dialogo para kay Gadamer ay isa palang interaksyon ng dalawang abot-tanaw (horizon) na kung magtatagumpay ay hahantong sa isang pagsasanib ng mga abot-tanaw (fusion of horizons), maaaninag na natin kung papaano iiral ang dialogo sa pagitan ng tao at ng teksto. Maiisip na natin sa puntong ito na isang dialogo ang hermenyutika sa pagitan ng mga abottanaw ng mambabasa at ng tekstong kanyang sinusuri. Dahil kahalintulad ang dialogo sa isang negosasyon, ang kalalabasan ng hermenyutika ay isang kahulugan na hindi subhektibo at hindi rin obhektibo. Para kay Gadamer, mas mahalaga palagi ang inter-subhektibong kahulugan. 


\section{Ang Parikala sa Likod ng Pamagat na Katotohanan at Pamamaraan}

Nabanggit na natin na ang hermenyutika ni Gadamer ay unang inilahad niya sa kanyang pangunahing librong Katotohanan at Pamamaraan. Ngunit ano nga ba para sa kanya ibig sabihin ng katotohanan at ng pamamaraan? Kapag ihahambing natin ang pamagat na ito sa kabuoang nilalaman ng obra, mararamdaman natin ang dalawang parikala (irony). Ang unang parikala (irony) ay nasa likod ng salitang "katotohanan" na sumasalungat sa pangkaraniwang ekspektasyon na ang parehong obra ay magtuturo sa sinumang babasa nito kung paano makakamtan ang katotohan. Subalit sa halip na ilahad niya ang katotohan na pangkaraniwang nalalaman ng pangkaraniwang mambabasa, ibang klaseng katotohanan pala ang tinutukoy ni Gadamer.

Malinaw na hindi sang-ayon si Gadamer sa isang subhektibong katotohanan, dahil katumbas lamang ito ng isang mambabasang nagpupumilit ipataw sa teksto ang kanyang sariling mga asumsyon at prekonsepsyon. Para kay Gadamer ang subhektibong katotohanan ay ang pangingibabaw ng isang bulag na prejudice. Hindi rin siya sang-ayon sa isang obhektibong katotohanan, dahil una, katulad ni Heidegger, hindi siya naniniwala na kayang matamo ng sinumang tao ang ganitong klase ng katotohanan; at pangalawa, nangangamba siya na ang obsesyon ng sangkatauhan para sa obhektibong katotohanan ay siyang ugat ng paghari-harian ngayon ng siyensya at teknolohiya na parehong sumisira sa kalikasan at sa isipan ng tao.

Para kay Gadamer ang katotohanan ay isang inter-subhektibo at eksistensyal na kalagayan na naka-ugat sa kasalukuyan nitong panahon at konteksto. Ibig sabihin, walang ni isang kalagayan na maaaring totooo sa lahat ng panahon at sa lahat ng konteksto. Higit pa sa obhektibong katotohanan, mas mahalaga para kay Gadamer ang pagtatanong at ang paghahanap ng mga kasagutan kahit pa man pansamantala lamang ang taglay na katotohan ng mga kasagutang ito. Sa konteksto ng dialogo, ang katotohanan ay yaong bagay na pinagkasunduan ng mga kasali sa dialogo na totoo. Sa sandaling kukupas na ang katotohanan ng bagay na ito, maaari itong pagtibayin muli o hindi kaya palitan ng bagong katotohanan sa pamamagitan ng panibagong dialogo.

Ang pangalawang parikala (irony) naman ay nasa likod ng salitang "pamamaraan" na sumasalungat sa pangkaraniwang ekspektasyon na ang parehong obra ay magtuturo sa sinumang mambabasa ng isang sistematiko at punto por puntong hermenyutika. Sa halip, determinado si Gadamer na ilayo ang proseso ng pag-uunawa at ng interpretasyon sa obsesyon ng Enlightenment tungkol sa obhektibo at teoretikal na usapin na palaging dapat nakabatay sa malilinaw, matatalas, mala-matimatikang pamamaraan. Naniniwala siya na ang paghahanap sa katotohanan ay isang inter-subhektibo at praktikal na gawain. 
Sa puntong ito, inihalintulad ni Gadamer ang proseso ng pag-uunawa at ng interpretasyon sa konseptong "phronesis" ni Aristoteles. Isang Griyegong salita ang "phronesis" na tumutukoy sa isang praktikal na siyensya na may kinalaman sa sitwasyunal, kontekstwal at moral na pagpapasya ng tao. Naniniwala si Gadamer na magkahawig ang moral na pagpapasya kung ano dapat ang ikikilos ng isang tao sa harap ng isang konteksto at sitwasyon at ang hermenyutikal na pagpapasaya kung ano dapat ang tamang kahulugan ng partikular na teksto. Tulad ng phronesis ang hermenyutikal na pagpapasya ay nagaganap lamang sa aktwal na proseso ng interpretasyon, at maliban sa kongkreto at eksistensyal na pagkakataong ito ang hermenyutikal na pagpapasya ay isa lamang walang laman na balangkas. Tulad din ng phronesis ang hermenyutikal na pagpapasya ay hindi rin maaaring ituro kagaya ng isang siyantipikong pamamaraan. Para kay Gadamer ang phronesis at ang hermenyutikal na pagpapasya ay maaaring maisasagawa at mapatalas lamang sa kongkreto at praktikal na paggamit lamang. Kahit alam ng isang tao ang heneral na pamantayang moral ng kanyang komunidad, hindi ito nangangahulugang gagawin niya palagi ang tama. Gayundin sa larangan ng hermenyutika, kahit alam ng isang mambabasa ang mga heneral na pamantayan ng proseso ng interpretasyon, hindi rin tio nangangahulugang magagawa niya palagi ang tamang interpretasyon. Kagaya na ang moral na pagpapasya ay maisasagawa ng tama at mapapatalas sa isang moral at matuwid na buhay, ang pag-uunawa at proseso ng interpretasyon ay maisasagawa ng tama at mapapatalas sa isang buhay na nakaalay sa pag-uunawa at proseso ng interpretasyon. Para kay Gadamer, ang phronesis, pag-uunawa at hermenyutika ay mga landas ng buhay na naisasagawa ng tama at napapatalas sa paulit-ulit na pagsasagawa.

Ang hermenyutika, sa puntong ito, ay mas malapit sa sining kaysa siyensya, at malapit sa sining ng buhay mismo kaysa anumang sining. Sa bandang huli ang obrang Katotohanan at Pamamaraan ay nagpahiwatig na na ang katotohanan ay hindi mahuhuli gamit ang anumang pamamaraan, dahil ang katotohanan ni Gadamer ay pamansamantalang maaaninag lamang gamit ang phronesis at dialogo.

\section{Mga Mahahalagang Puntos ng Dialektikal na Hermenyutika para sa Ating Lokal na Pag-aaral ng Teksto at Kultura}

May iilang mahalagang puntos ang makukuha natin mula sa hermenyutika nina Heidegger, Bultmann at Gadamer na magagamit natin sa pagpapayaman at pagpapayabong sa sarili nating tekstuwal at kultural na mga proyekto, lalong-lalo na sa larangan ng araling Pilipino.

Unang-una sa mga ito ay ang kahalagahan sa ginawa ni Heidegger na pagkakaiba sa pagitan ng proseso ng pag-uunawa at ng interpretasyon. Nagpapahiwatig ito sa atin ng isang pamantayan na ang isang mambabasa, o 
iskolar, ay kailangang magkakaroon muna ng mayaman at malalim na kaalaman bago pa man siya sasabak sa isang hermenyutikal na gawain. Para kay Heidegger, kahit pa man inter-subhektibo ang proseso ng interpretasyon, hindi ito kayang isasagawa ng sino-sino na lamang, dahil tanging mga taong may kasanayan na at nakapagpatalas na ng kanilang mga pasiunang balangkas sa pag-uunawa ang handa para nito. Ibig sabihin, ang mahusay na mambabasa, o iskolar, ay hindi iyong nagsisimula pa lamang sa larangan, kung hindi iyong may mahaba-haba na, o hindi kaya may masinsinan na, na karasanasan sa pakikitungo sa mga teksto. Isang implikasyon sa ginawang pagkakaiba ni Heidegger sa pagitan ng pag-uunawa at interpretasyon ay ang isa pang pamantayan na habang nagiging mas kumplikado at mas teknikal ang isang tekstwal na proyekto, dapat ding magiging mas mayayaman, mas malalalim, at mas matatalas ang mga pasiunang balangkas ng pag-uunawa ng mambabasa, o iskolar.

Pangalawa sa mga ito ay ang pagpuksa ni Heidegger sa obhektisismong nanghihimasok at naghari-harian na sa mga larangan ng hermenyutika, humanidades at agham panlipunan. Niligtas ni Heidegger ang mga mambabasa, o iskolar, mula sa walang saysay at labis na pag-aabala kung papaano mapapanatili ang pagiging obhektibo. Ang panahon at lakas na matitipid mula dito ay maaaring ilaan na lamang ng mambabasa, o iskolar, sa pagpapayaman at pagpapalalim ng kanyang pananaliksik. Subalit, importante na mauunawaan din natin na ang pagpapahalaga ni Heidegger sa inter-subhektibong kahulugan ay hinding-hindi nagbubukas ng isang landas para sa mga mambabasa, o iskolar, na tinatamad mananaliksik at gusto lamang makagawa ng madaling interpretasyon. Alalahanin natin palagi na sina Heidegger, Bultmann at Gadamer, kahit hindi sila naniniwala sa obhektibong kahulugan, ay puro mga masisinop at pulidong iskolar.

Pangatlo sa mga ito ay ang mga kaisipang makukuha natin mula sa inilahad na kritisismo ng porma ni Bultmann, kahit sa istriktong usapan ay hindi ito saklaw ng dialohikal na hermenyutika. Maaari nating ilabas sa biblikal na konteksto ang kritisismo ng porma at ipataw sa ating mga kultural na teksto. Ang ating mga epiko ay maaaring himayin gamit ang pamamaraang ito para lalo nating silang maiintindihan. Ang ating mga nakasulat na panitkan, kung saan nagkahalohalo na ang mga katutubo, Espanyol at Amerikanong salik ay magandang gamitan din ng kritisismo ng porma. Maging ang arkitektura ng ating mga simbahan at bahay na bato, kung saan maaaninag pa rin natin ang katutubo, Tsino, Espanyol, Mehikano, at Muslim na mga elemento, ay mas maiintindihan natin kung susuriin sila gamit ang kritisismo ng porma. Napakaraming hermenyutikal na proyekto sa araling Pilipino ang maaaring madalumat gamit ang kritisismo ng porma ni Bultmann.

Pang-apat sa mga ito ay ang pahayag ni Bultmann, na sinang-ayunan naman ni Gadamer, na walang opsyon ang isang mambabasa, o iskolar, kung hindi dalhin ang kanyang mga katanungan at paksang pinagkaka-abalahan sa kanyang 
pananaliksik. Kung wala ang mga mental na bagaheng ito, wala rin namang sapat na motibo ang mambabasa, o iskolar, na magtitiyaga sa paghalungkat at pagbasa ng santambak na mga teksto. Ang nasyunalistikong sentimiyento na ngayon ay masigasig na nagpapakilos sa ating araling Pilipino ay isang halimbawa ng mga mental na bagaheng ito. Kaya madaling sitahin ang kasalukuyan nating araling Pilipino ng mga makikitid na tagapagtaguyod ng obhektisismo na ito raw ay dispalinghadong pananaliksik. Ang proyekto ng kasalukuyan nating araling Pilipino na labanan at batikusin ang mga kanluraning kaalaman at pananaliksik tungkol sa ating bayan ay maaaring huhugot ng epistemolohikal na lehetimasyon mula sa kaisipan nina Bultmann at Gadamer.

Panglima sa mga ito ay ang mga kaisipang makukuha natin mula sa inilahad na demitolohisasyon ni Bultmann, kahit sa istriktong usapan ay hindi rin ito saklaw ng dialohikal na hermenyutika. Kahit pa man hindi kanais-nais at katanggap-tanggap ang isang teksto, maaari pa rin itong magtataglay ng mahahalagang mga mensahe at kaalaman. Ang mga teksto halimbawa na naisulat ng diktador na si Ferdinand Marcos at ng kanyang mga nabayarang intelektwal ay labis nang nakukulayan sa mga kasamaan at pang-aabusong naganap ng kanyang rehimen. Ngunit ang pamamaraan at teorya ng demitolohisasyon ay nagtuturo sa atin na kahit ayaw nating malapitan man lamang ang mga tekstong ito, hindi imposible na mayroong silang mga nakakubling mahahalagang diskurso tungkol sa nasyunalismo at mga mungkahi kung papaano patatagin ang estado ng Pilipinas.

Pang-anim sa mga ito ay ang pagbibigay diin ni Gadamer na ang hermenyutika ay isang landas ng buhay. Sa kaisipan ni Gadamer, ito ay dahil ang proseso ng interpretasyon ay kahalintulad ng phronesis ni Aristoteles na nagiging mas mahusay at mas matalas habang paulit-ulit itong isinasagawa. Kaya ang isang mambabasa, o iskolar, ay hindi rinng maaaring mambabasa, o iskolar, lamang sa taong ito, at sa susunod na taon ay hindi muna, para lamang bumalik ulit sa paglipas ng dalawa o tatlong taon. Para kay Gadamer ang hermenyutika ay isa ring seryosong bokasyon, o landas ng buhay, para sa mga seryosong mambabasa, o iskolar.

Department of Filipino, De La Salle University-Manila, Philippines

\section{References}

Heidegger, Martin, Being and Time (New York: Harper and Row, 1962).

Caputo, John, Radical Hermeneutics: Repetition, Deconstruction, and the Hermeneutic Project (Bloomington: Indiana University Press, 1987).

Beyer, Christian, "Edmund Husserl," sa Stanford Encyclopedia of Philosophy, $<$ http://plato.stanford.edu/entries/husserl/\#EmpIntLif $>$ Accessed: August 2, 2012.

(C) 2012 F.P.A. Demeterio

http://www.kritike.org/journal/issue 11/demeterio june2012.pdf

ISSN 1908-7330 
Demeterio, Feorillo, "Ang Hermenyutika nina Schleiermacher at Dilthey bilang Batayang Teoretikal sa Araling Pilipino,” sa Kritike, 5:1 (June 2011).

Bultmann, Rudolf, "The New Approach to the Synoptic Problem," sa Ogden, Schubert, Existence and Faith: Shorter Writings by Rudolf Bultmann (London: Fontana Library, 1964).

Bultmann, Rudolf, "Is Exegesis without Presupposition Possible," sa Ogden, Schubert, Existence and Faith: Shorter Writings by Rudolf Bultmann (London: Fontana Library, 1964).

Gadamer, Hans-Georg, Truth and Method (New York: Continuum Publishing Company, 1999). 